\title{
NONGENERATORS OF RINGS
}

HOMER BECHTELL

The purpose of this note is to examine the role of nongenerators in the theory of rings, i.e. the elements $x$ of a ring $R$ such that for each subset $M$ of $R$ for which $R=\langle x, M\rangle$, then $\langle M\rangle=R$. The approach used considers a ring as a group with multiple operators and consequently an ideal $A$ generated by a subset $S$ implies that $S \subseteq A$. These results will include those of L. Fuchs [1] and A. Kertesz [2] whenever the ring has unity.

Unless otherwise indicated, the terminology and the necessary known results may be found in N. McCoy's text [3].

Denote the ideal (right ideal) generated by the set $M$ of $R$ by $\langle M\rangle$ $\left(\langle M\rangle_{r}\right)$.

Definition. An element $x \in A$ is a generator of an ideal (right ideal) $A$ in a ring $R$ provided that there is a subset $M$ of $A$ such that $A=\langle x, M\rangle\left(A=\langle x, M\rangle_{r}\right)$ and $\langle M\rangle \subset A\left(\langle M\rangle_{r} \subset A\right)$ properly. Otherwise $x$ is called a nongenerator of $A$. (Note that $M$ may be empty.)

The set of nongenerators of an ideal (right ideal) $A$ in a ring $R$ will be denoted by $\Phi\left(\Phi_{r}\right)$, respectively.

Immediate consequences of the definition are the following:

(i) For an element $x$ of a ring $R, x \in \Phi\left(x \in \Phi_{r}\right)$ if and only if $\langle x\rangle \subseteq \Phi$ $\left(\langle x\rangle_{r} \subseteq \Phi_{r}\right)$.

(ii) In a ring $R, \Phi$ is an ideal and $\Phi_{r}$ is a right ideal.

Throughout this paper a maximal ideal of a ring $R$ will be a proper ideal of $R$ that is not contained in another proper ideal of $R$. Similarly for maximal right (left) ideals.

(iii) In a ring $R, \Phi\left(\Phi_{r}\right)$ is the intersection of the maximal ideals (right ideals), if they exist, and is $R$ otherwise.

(iv) For a ring $R$ and homomorphism $\theta$ of $R, \Phi \theta \subseteq \Phi(R \theta)$ and $\Phi_{r} \theta \subseteq \Phi_{r}(R \theta)$.

(v) For an ideal $A$ of a ring $R, A \subseteq \Phi$ implies that $\Phi(R / A)=\Phi / A$ and $A \subseteq \Phi_{r}$ implies that $\Phi_{r}(R / A)=\Phi_{r} / A$.

(vi) For a ring $R$, if $A$ is an ideal (right ideal) of $R$, then $\Phi(A) \subseteq \Phi$ $\left(\Phi_{r}(A) \subseteq \Phi_{r}\right)$.

(vii) In a ring $R, \Phi=(0) \quad\left(\Phi_{r}=(0)\right)$ implies that $\Phi(A)=(0)$ $\left(\Phi_{r}(A)=(0)\right)$ for each ideal (right ideal) $A$ of $R$. Such rings will be called $\Phi$-free or $\Phi_{r}$-free respectively.

Received by the editors December 4, 1966. 
(viii) In a ring $R, A=\Phi(A)\left(A=\Phi_{r}(A)\right)$ for an ideal (right ideal) $A$ of $R$ implies that $A \subseteq \Phi\left(A \subseteq \Phi_{r}\right)$.

(ix) If $R$ is a ring and $R=M_{1} \oplus \cdots \oplus M_{n}$, then $\Phi=\Phi\left(M_{1}\right) \oplus \cdots$ $\oplus \Phi\left(M_{n}\right)$ for ideals $M_{i}$ of $R$.

(x) In a ring $R$, if $A$ is a minimal ideal (right ideal) such that $A \Phi \Phi\left(A \Phi \Phi_{r}\right)$, then there exists a maximal ideal (right ideal) $B$ such that $R=A \oplus B$.

(xi) If $R$ is a zero ring $\left(R^{2}=(0)\right)$, then $\Phi=\Phi\left(R^{+}\right), \Phi\left(R^{+}\right)$the Frattini subgroup of the additive group $R^{+}$.

In the remaining portion of this note, the Jacobson radical and the upper Baer radical will be denoted by $J$ and $N$ respectively.

TheOREM 1. In a ring $R, \Phi_{r} \subseteq J$ and $\Phi \subseteq N$.

Proof. If $J \neq R$, then $J$ is the intersection of the modular maximal right ideals of $R$; and if $N \neq R$, then $N$ is the intersection of the modular maximal ideals.

Note that in Theorem 1 equality may not occur as the ring $\{0,2 ; \bmod 4\}$ exemplifies.

THEOREM 2. In a ring $R, R J \subseteq \Phi_{r}$ and $J R \subseteq \Phi_{l}, \Phi_{l}$ denoting the set of nongenerators with respect to left ideals.

Proof. Since the result follows if $\Phi_{r}=R$, consider the case that $\Phi_{r} \subset R$ properly. Suppose there is an element $x \in R$ such that $y x \notin \Phi_{r}$ for some element $y \in R$. Then there exists a maximal right ideal $M$ such that $y x \notin M$. $M$ defines a simple right $R$-module $R / M \cong R^{*}$, and under the natural $R$-homomorphism $\theta$ of $R \rightarrow R^{*}, y \theta \neq 0$ and $(y x) \theta \neq 0$. So $\left(R^{*}\right) R=R^{*}$, and an element $z \in R$ exists such that $(y x z) \theta=y \theta$. Then note that if $x z$ is r.q.r., there exists an element $b \in R$ such that $x z+b=x z b$. Under $\theta, \quad y x z+y b=y x z b$ becomes $(y x z) \theta=-(y b) \theta$ $+(y x z b) \theta=-(y b) \theta+(y b) \theta=0$. So $y x z \in M$ and a contradiction. Therefore $x z$ cannot be r.q.r. In conclusion, if $x$ has the property that $y x \notin \Phi_{r}$ for some $y \in R$, then $x \notin J$. So for each element $x \in J, R x \subseteq \Phi_{r}$, i.e. $R J \subseteq \Phi_{r}$. Similarly $J R \subseteq \Phi_{l}$. (Note: this proof was suggested by a result of Kertesz [2].)

COROllaRy 2.1. (a) For a ring $R, \Phi_{r}$ and $\Phi_{l}$ are ideals in $R$.

(b) For a ring $R, J^{2} \subseteq \Phi_{r} \cap \Phi_{l}$.

(c) $J=\left(\Phi_{r}: R\right)=\left(\Phi_{l}: R\right)$

(d) For a ring $R, x \in J$ iff $R \times R \subseteq \Phi_{r} \cap \Phi_{l}$.

Since in general both $\Phi_{r}$ and $\Phi_{l}$ are in $J$, it follows that in each primitive ring the right ideals and the left ideals are $\Phi_{r^{-}}$and $\Phi_{l}$-free respectively. If the ring is a simple nonradical ring, then the ring is 
$\Phi$-free. For the simple primitive rings, all three hold. And for a field $F, \Phi(F)=(0)$.

In general $\Phi \Phi J$. For example: let $R$ be the ring of all linear transformations of a vector space $V$ with a denumerable basis. It is known (e.g., see [3]) that $R$ is a primitive ring and $J=(0)$. Since $R$ has unity, $N=R$; and, in fact, the only proper ideal besides (0) is the ideal of elements of finite rank. This ideal is $N=\Phi$. Also note that $\Phi_{r}=\Phi_{l}=(0)$.

Theorem 3. For a ring $R$ having $R^{2}=R, \Phi$ is a semiprime ideal.

Proof. Each maximal ideal is prime. If $A$ is an ideal for which $A^{2} \subseteq \Phi$, then $A^{2}$ is contained in each maximal ideal $M$. So $A$ is contained in each $M$. Therefore $A \subseteq \Phi$.

CoROllaRy 3.1. For a ring $R$ having $R^{2}=R$, the prime radical is contained in $\Phi$.

CoROllaRY 3.2. For a ring $R$ having $R^{2}=R, J \subseteq \Phi$ iff $J^{2} \subseteq \Phi$.

TheOREM 4. For a ring $R$ having $R^{2}=R$ and center $Z, N \cap Z \subseteq \Phi_{r}$ and $N \cap Z \subseteq \Phi$.

Proof. If $A=N \cap Z \Phi \Phi_{r}$ and $M$ is a maximal right ideal not containing $A$, then $R=A+M$. This implies that $M$ is a maximal ideal, and $R^{2}=R$ implies that $R / M$ is a simple commutative nonzero ring. Hence $M$ is modular and $N \subseteq M$ implies that $A \subseteq M$. So $A \subseteq \Phi_{r}$. Similarly $N \cap Z \subseteq \Phi$.

Corollary 4.1. If $R$ is a commutative ring and $R^{2}=R$, then $J=\Phi$.

Theorem 5. For a ring $R$ having $R^{2}=R, \Phi_{r}=\Phi_{l}=J$.

Proof. Consider $\Phi_{r}$ and note that for $R=\Phi_{r}$ and $\Phi_{r} \subseteq J$ implies that $J=\Phi_{r}$. So then consider the case that $\Phi_{r} \subset R$ properly. By Theorem 2, $J^{2} \subseteq \Phi_{r}$. Form $R / J^{2} \cong R^{*}$ noting that $J^{*} \cong J\left(R / J^{2}\right)=J / J^{2}$ and that $\Phi_{r}^{*} \cong \Phi_{r}\left(R / J^{2}\right)=\Phi_{r} / J^{2}$. If $x \in J^{*}$ and $x \notin \Phi_{r}^{*}$, there exists a maximal right ideal $M^{*}$ such that $x \notin M^{*}$. Under the natural $R^{*}$-homomorphism $\theta$ of $R^{*} \rightarrow R^{*} / M^{*}, R^{*}$ is mapped on to a simple right $R^{*}$-module $R^{*} / M^{*}$. Since $x \notin M^{*}$, then $J^{*} \theta=R^{*} / M^{*}$. But $J^{* 2} \theta=(0)$ implies that $R^{*} / M^{*}$ is annihilated by $R^{*}$, i.e. $\left(R^{*} / M^{*}\right) R^{*}=(0)$. This contradicts the hypothesis that $R^{2}=R$ since, in turn, this implies that $R^{* 2}=R^{*}$ and $\left(R^{*} / M^{*}\right) R^{*}=R^{*} / M^{*}$. So $J^{*} \subseteq M^{*}$. This leads to $J^{*} \subseteq \Phi_{r}^{*}$ and hence $J \subseteq \Phi_{r}$. So the result follows.

Similarly $\Phi_{l}=J$.

CoRollary 5.1 (L. Fuchs [1]). For a ring with unity, $\Phi_{r}=\Phi_{l}=J$. 
THEOREM 6. If $R$ satisfies the d.c.c. on right ideals, then $\Phi=(0)$ if and only if $R$ is a direct sum of a finite collection of simple ideals.

Proof. Consider the intersections of all finite collections of maximal ideals. By the d.c.c. on right ideals, each linear system has a minimal element, say $D$. If $M$ is a maximal ideal, then $D=D \cap M$. So $D \subseteq \Phi$ and $D=(0)$. As is known, if there exists in a ring a finite number of maximal ideals $M_{i}(i=1, \cdots, n)$ with zero intersection, then $R$ is isomorphic to the direct sum of some or all the simple rings $R / M_{i}(i=1, \cdots, n)$. By (ix) each direct summand has $\Phi\left(R / M_{i}\right)=(0)$ since $R / M_{i}$ is a simple ideal.

Again by (ix) the converse is evident.

TheORem 7. If $R$ is a ring with d.c.c. on right ideals, then both $\Phi_{r}$ and $\Phi_{l}$ are contained in $\Phi$.

Proof. The theorem is valid whenever $R=\Phi$, so consider the case that $\Phi \subset R$ properly. In particular restrict attention to $R^{*}=R / M$ for a maximal ideal $M$. For either $R^{* 2}=(0)$ or $R^{* 2}=R^{*}, \Phi_{r}^{*}=(0)$. Hence under the natural homomorphism $\theta$ of $R \rightarrow R^{*}, \Phi_{r} \theta \subseteq(0)$ implies that $\Phi_{r} \subseteq M$. So $\Phi_{r} \subseteq \Phi$ and similarly $\Phi_{l} \subseteq \Phi$.

ThEOREM 8. If $R$ is a ring with the d.c.c. on right ideals, then $\Phi_{r}$ $=\Phi_{l}=\Phi$.

Proof. Since $\Phi_{r}$ is an ideal of $R$ form $R^{*} \cong R / \Phi_{r}$ having $\Phi_{r}^{*}$ $=\Phi_{r}\left(R^{*}\right)=(0), \Phi^{*} \cong \Phi / \Phi_{r}$ and $J^{*} \cong J / \Phi_{r}$. If $M^{*}$ is a maximal right ideal such that $\Phi^{*} \Phi M^{*}$, then $R^{*}=\Phi^{*}+M^{*}$. However, since $R^{*} J^{*}$ $\subseteq \Phi_{r}^{*}=(0)$, then $\Phi^{*}$ is in the annihilator of $M^{*}$. This implies that $M^{*}$ is an ideal of $R^{*}$ and hence a contradiction to the assumption that $\Phi^{*} \subseteq M^{*}$. So $\Phi^{*}=(0)$, i.e. $\Phi \subseteq \Phi_{r}$, and the result follows. Similarly, $\Phi_{l}=\Phi$.

THEOREM 9. For a ring $R$ with d.c.c. on right ideals and $R$ not a radical ring, then $\Phi=J$ if and only if $R^{2}=R$.

Proof. Suppose $R^{2}=R$ and there exists a maximal ideal $M$ such that $J \Phi M$. Then under the natural homomorphism $\theta$ of $R \rightarrow R / M$ $=R^{*}, J \theta=R^{*}$. However, since $J^{2} \subseteq \Phi \subseteq M$ it follows that $R^{* 2}=(0)$ and this contradicts $R^{2}=R$. So $J \subseteq \Phi$. Since $J=N$ and $\Phi \subseteq N$, then $\Phi=J$.

On the other hand, suppose that $J=\Phi \subset R$ properly. Form $R / \Phi$ $\cong R^{*}$ and note that $J^{*} \cong J(R / \Phi)=(0)=\Phi^{*} \cong \Phi(R / \Phi)$. As is known, $J^{*}=(0)$ implies that $R^{* 2}=R^{*}$. If $R^{2} \subset R$ properly and $R^{2} \theta=R^{*}$ under the natural homomorphism $\theta$ of $R \rightarrow R^{*}$, then $R=\Phi+R^{2}=R^{2}$ and a contradiction. So $R^{2}=R$. 
In a radical ring $R$ the condition $\Phi=J$ does not necessarily imply that $R^{2}=R$. For example, let $R$ be a zero ring having $R^{+}$a group of type $p^{\infty}$. Then $\Phi(R)=\Phi\left(R^{+}\right)=R^{+}$and $J=R$.

\section{BIBLIOGRAPHY}

1. L. Fuchs, A remark on the Jacobson radical, Acta Sci. Math (Szeged) 14 (1952), 167-168.

2. A Kertesz, A characterization of the Jacobson radical, Proc. Amer. Math. Soc. 14 (1963), 595-597.

3. N. McCoy, The theory of rings, Macmillan, New York, 1964.

UNIVERSITY OF NEW HAMPSHIRE 